\title{
Design and Fabrication Highlights Enabling a 2 mm, 128 Element Bolometer Array for GISMO
}

Christine A Allena. Dominic J Benford ${ }^{b}$. Timothy M Miller ${ }^{a, 0}$. Johannes G Staguhn ${ }^{b, d}$. Edward J Wollack ${ }^{b}$. S. Harvey Moseley

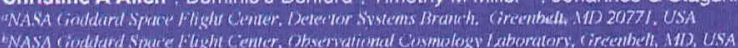

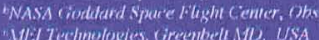

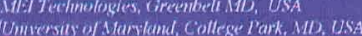

- Goal: Design and fabrication of a background limited, 128 pixel Tran-sition Edge Sensor (TES) bolometer array for the Goddard IRAM Super-conducting 2-mm Observer (GISMO)
-Design approach: The array design is based on our BUG (Backshort Under Grid) detector architecture; a square grid array fabricated on $1.4 \mu \mathrm{m}$ SiliconOn-Insulator (SOI) wafers with $1 / 4$ wavelength resonance backshort, optimized for $2 \mathrm{~mm}$
- Result: The GISMO array, having $8 \times 16$ pixels on a 2 $\mathrm{mm}$ pitch, covers an area $16 \times 32 \mathrm{~mm}$. The bolometers are $1.4 \mu \mathrm{m}$ thick and fabricated on the top layer of SOI wafers. The $500 \mu \mathrm{m}$ handle wafer is etched into a square grid to support the bolometer array and provide the $1 / 4$ wavelength backshort spacing. The backshort is a reflective copper coating plated onto the alumina detector board.

\section{GISMO Array Design}

- Detector for 2-millimeter radiation fabricated on $1.4 \mu \mathrm{m}$ SOI wafers with $500 u m$ thick supporting square grid. The array is mounted on a copper plated alumina board, the copper coating acts as an optical reflector.

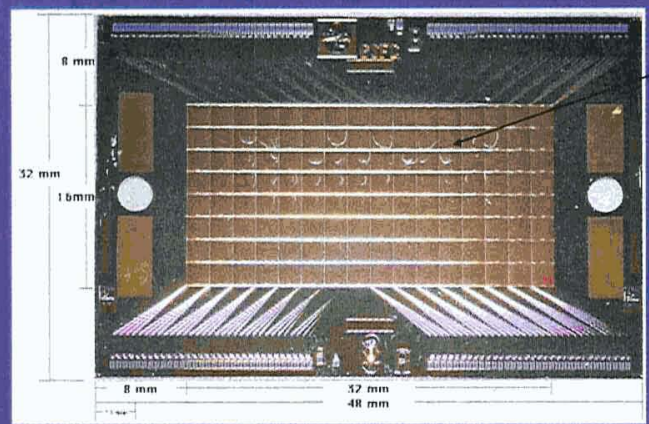

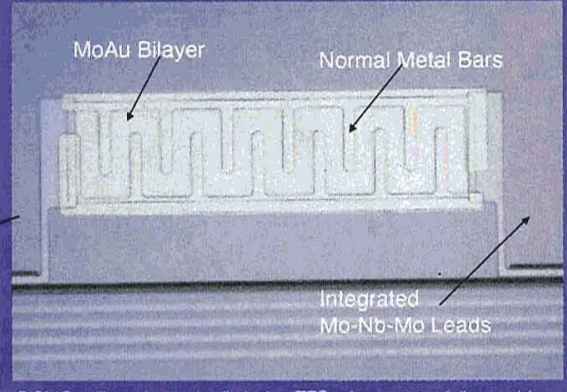

GISMO utilizes the "zebra" design TES, also successfully used for Goddard's FIBRE instrument at CSO. The design incorporates a relatively thick gold film covering the sensors edges and extending $2 / 3$ of the distance across the sensor in an interdigitated comb design. This design significantly suppresses out-of-band $1 / f$ noise in the sensor.
Fabrication sequence:

-Batch sputter-sputter Mo-Au deposition Dry etch gold TES

Sputter Nb-Mo leads (enhance critical current) - Dry etch Mo-Nb-Mo leads

Lift-off normal metal gold zebra stripes Lift-off aluminum bonding pads

-Mask detector body on $1.4 \mu \mathrm{m}$ Sol top layer Wax water to temporary backing water Mask delector square grid from back-side - Deep Reactive lon Elch square grid through $500 \mu \mathrm{m}$ handle wate

Release from wax

Reactive lon Eich detector body on front side Deposit bismuth $400 \Omega$ absorber on back-side of detector

\section{Detector Characterization}

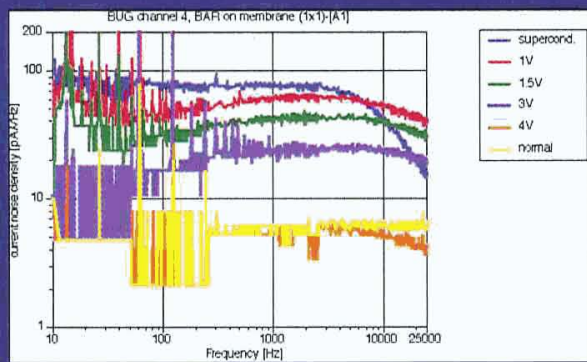

Frequency dependent noise of BUG detector with Zebra design normal metal bars. The noise levels in the region of operation are well within the desired target to permit background limited detector performance

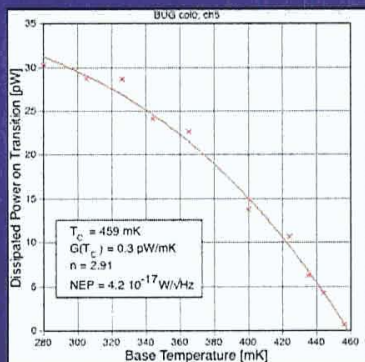

Detectors shown, have a measured thermal conductance $G=0.3 \mathrm{pW} / \mathrm{mK}$ (a) $460 \mathrm{mK}$
- GISMO will be operated at the IRAM $30 \mathrm{~m}$ telescope at Pico Veleta, Spain, during the summer months.

$\cdot 150 \mathrm{GHz}$, Sky Noise $=1.6 \times 10^{-16} \mathrm{~W} / \sqrt{\mathrm{Hz}}$ - GISMO $2 \mathrm{~mm}$ pixel NEP $=\sim 4 \times 10^{-17} \mathrm{~W} / \sqrt{\mathrm{Hz}}$
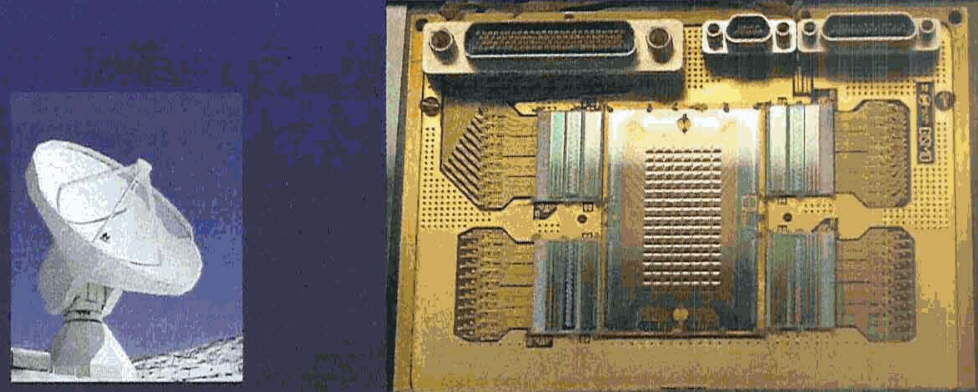

The GISMO array seen here fully populated with four columns of NIST $1 \times 32$ SQUIDs, Nyquists, and Goddard shunt resistors. The array is mounted on a copper-plated alumina board, fastened to flexures machined into the base of the gold-plated copper detector flexures machined into the base of the gold-plated copper cetector detector board. 